\title{
Categorical agreements and discrepancies of direct susceptibility tests in Acinetobacter from blood cultures
}

Bacteraemia is a clinically critical condition and needs a prompt intervention that includes the administration of antibiotics. About 30 years ago it was demonstrated that results from direct susceptibility tests (DSTs) using agar diffusion (AD) with the broth of a positive blood culture were comparable to those obtained by the conventional method (Mirrett \& Reller, 1979; Doern et al., 1981). Since then, different evaluations of this alternative procedure were carried out, including those that used automated systems (Bruins et al., 2004; Quesada et al., 2010; Wellinghausen et al., 2007). The emergence of organisms presenting different mechanisms of antimicrobial resistance, including multidrug-resistant Acinetobacter, was prominent during recent decades and changed the susceptibility tests carried out in the clinical laboratory. In Brazil, multidrugresistant Acinetobacter is becoming common in many hospitals as a cause of bacteraemia (Prates et al., 2011).

Therefore, we investigated the performance of DSTs using AD in blood cultures (BacT/ALERT FA; bioMérieux) containing Gram-negative bacilli from patients at the Hospital Mãe de Deus, Porto Alegre, Brazil, between December 2007 and November 2009. Positive blood cultures containing Gram-negative bacilli were subjected to DSTs using AD: an aliquot of $50 \mu$ l blood-broth mixture was spread on the surface of Mueller-Hinton agar plates (Oxoid) and the remaining steps of the procedure were carried out according to the guidelines of the Clinical and Laboratory Standards Institute (CLSI, 2008). All isolates were submitted to the reference $\mathrm{AD}$ test after subculture on solid media. Results of DSTs using AD and conventional $\mathrm{AD}$ tests were compared in terms of categorical agreements and discrepancies: minor (Mi), a change involving an intermediate result; major (Ma), a resistant result by
DSTs and a susceptible result by conventional $\mathrm{AD}$; and very major (VM), a susceptible result by DSTs in a resistant organism as defined by conventional AD (Doern et al., 1981).

In 136 blood cultures growth of a Gramnegative bacillus was observed. In 99 blood cultures a member of the family Enterobacteriaceae was identified. Amongst the non-fermentative Gram-negative bacilli, we identified Pseudomonas aeruginosa (19) and Acinetobacter (Acinetobacter baumannii= 17 and Acinetobacter lwoffii=1). All Acinetobacter isolates were hospital acquired and considered cases of true bacteraemia. Overall agreement with the reference method, including 1131 antibiotic/ organism combinations, was $82.3 \%$ (931/ 1131), with $9.90 \%$ (112/1131), $6.37 \%$ (72/ $1131)$ and $1.41 \%(16 / 1131) \mathrm{Mi}, \mathrm{Ma}$ and VM errors, respectively. For Acinetobacter, however, agreement was $73.8 \%$ (93/126 antibiotic-organism combinations), with $\mathrm{Mi}=8.73 \%, \mathrm{Ma}=13.5 \%$ and $\mathrm{VM}=3.97 \%$. Details for the different antimicrobials are shown in Table 1. The resistance rates of the Acinetobacter isolates to the different antimicrobial agents, according to CLSI guidelines were: $38.9 \%(7 / 18)$ to amikacin and gentamicin, $55.6 \%(10 / 18)$ to

meropenem and piperacillin/tazobactam, $61.1 \%(11 / 18)$ to cefepime, $72.2 \%(13 / 18)$ to ciprofloxacin, and $72.2 \%(13 / 18)$ to trimethoprim/sulphamethoxazole. Three additional isolates exhibited intermediate resistance to amikacin $(n=2)$ or piperacillin/tazobactam $(n=1)$. We did not perform tests for colistin or tigecycline.

Bacteraemias due to Acinetobacter used to be rare; however, this organism is increasing its importance worldwide (Peleg et al., 2008). Studies that measured accuracy of DSTs from positive blood cultures so far did not include a relevant number of Acinetobacter isolates. To the best of our knowledge, this is the first study with a considerable proportion of Acinetobacter isolates (13.2\% of all Gramnegative bacilli), and clearly shows that DSTs are not adequate for Acinetobacter, especially for some antibiotics such as amikacin, cefepime, gentamicin, piperacillin/tazobactam and trimethoprim/sulphamethoxazole. Also, VM errors were frequent with aminoglycosides. Moreover, false resistance (Ma errors) was observed for all agents, and results of DSTs may thus limit therapeutic options to treat Acinetobacter bacteraemia. We recommend a cautious

Table 1. Categorical agreements and discrepancies of DSTs amongst 18 Acinetobacter from blood cultures (126 antibiotic/organism combinations)

\begin{tabular}{|lcccc|}
\hline \multirow{2}{*}{ Antimicrobial agent } & \multicolumn{4}{l}{ No. of categorical agreements and discrepancies (\%) } \\
\cline { 2 - 5 } & Agreement & Mi error & Ma error & VM error \\
\hline Amikacin & $8(44.4)$ & $4(22.2)$ & $2(11.1)$ & $4(22.2)$ \\
Cefepime & $11(61.1)$ & $4(22.2)$ & $3(16.7)$ & 0 \\
Ciprofloxacin & $16(88.9)$ & $1(5.56)$ & $1(5.56)$ & 0 \\
Gentamicin & $13(72.2)$ & 0 & $4(22.2)$ & $1(5.56)$ \\
Meropenem & $16(88.9)$ & 0 & $2(11.1)$ & 0 \\
Piperacillin/tazobactam & $13(72.2)$ & $2(11.1)$ & $3(16.7)$ & 0 \\
Trimethoprim/sulphamethoxazole & $16(88.9)$ & 0 & $2(11.1)$ & 0 \\
Total & $93(73.8)$ & $11(8.73)$ & $17(13.5)$ & $5(3.97)$ \\
\hline
\end{tabular}


use of DSTs in hospitals with bacteraemia due to Acinetobacter.

\section{Vinicius Sgorla Couto, ${ }^{1}$ Caroline Reis de Barros, ${ }^{1}$ Ana Carolina Sayão Lobato Cerri, ${ }^{1}$ Leandro Reus Rodrigues Perez ${ }^{2}$ and Cícero Armidio Gomes Dias ${ }^{1}$}

${ }^{1}$ Universidade Federal de Ciências da Saúde de Porto Alegre, Rua Sarmento Leite 245, Porto Alegre, Brazil

${ }^{2}$ Hospital Mãe de Deus, Porto Alegre, Brazil

Correspondence:

Cícero Armidio Gomes Dias

(cicero.dias@pq.cnpq.br)

Bruins, M. J., Bloembergen, P., Ruijs, G. J. H. \& Wolfhagen, M. J. H. M. (2004). Identification and susceptibility testing of Enterobacteriaceae and Pseudomonas aeruginosa by direct inoculation from positive BACTEC blood culture bottles into Vitek 2. J Clin Microbiol 42, 7-11.

CLSI (2008). Performance Standards for Antimicrobial Susceptibility Testing, 18th informational supplement, M100-S18. Wayne, PA: Clinical and Laboratory Standards Institute.

Doern, G. V., Scott, D. R., Rashad, A. L. \& Kim, K. S. (1981). Evaluation of a direct blood culture disk diffusion antimicrobial susceptibility test. Antimicrob Agents Chemother 20, 696-698.

Mirrett, S. \& Reller, L. B. (1979). Comparison of direct and standard antimicrobial disk susceptibility testing for bacteria isolated from blood. J Clin Microbiol 10, 482-487.

Peleg, A. Y., Seifert, H. \& Paterson, D. L. (2008). Acinetobacter baumannii: emergence of a successful pathogen. Clin Microbiol Rev 21, 538582.
Prates, C. G., Martins, A. F., Superti, S. V., Flores, F. S., Ramos, F., Cantarelli, V. V. \& Zavascki, A. P. (2011). Risk factors for 30-day mortality in patients with carbapenem-resistant Acinetobacter baumannii during an outbreak in an intensive care unit. Epidemiol Infect 139, 411-418.

Quesada, M. D., Giménez, M., Molinos, S., Fernández, G., Sánchez, M. D., Rivelo, R., Ramírez, A., Banqué, G. \& Ausina, V. (2010). Performance of VITEK-2 Compact and overnight MicroScan panels for direct identification and susceptibility testing of Gramnegative bacilli from positive FAN BacT/ALERT blood culture bottles. Clin Microbiol Infect 16, 137-140.

Wellinghausen, N., Pietzcker, T., Poppert, S., Belak, S., Fieser, N., Bartel, M. \& Essig, A. (2007). Evaluation of the Merlin MICRONAUT system for rapid direct susceptibility testing of gram-positive cocci and gram-negative bacilli from positive blood cultures. J Clin Microbiol 45, 789-795. 